\title{
Time-resolved and Temperature-dependent Broadband Emission of Plasmon-coupled Quantum Dots
}

\author{
Quinton Rice ${ }^{1,2}$, Sangram Raut ${ }^{3}$, Raul Chib ${ }^{3}$, Zygmunt Gryczynski ${ }^{3,4}$, Ignacy Gryczynski³, \\ Andrew Wang ${ }^{5}$, Willliam Y. Yu ${ }^{6}$, Bagher Tabibi ${ }^{1}$, Felix Jaetae Seo ${ }^{1}$ \\ 1. Advanced Center for Laser Science and Spectroscopy, Department of Physics, Hampton University, Hampton, \\ Virginia 23668, USA \\ 2. Department of Chemistry and Physics, The University of North Carolina at Pembroke, Pembroke, NC 28372, \\ USA \\ 3. Center for Fluorescence Technologies and Nanomedicine, Department of Cell Biology and Immunology, \\ University of North Texas Health Science Center, Fort Worth, Texas 76107, USA \\ 4. Department of Physics and Astronomy, Texas Christian University, Fort Worth, Texas, 76129, USA \\ 5. Ocean NanoTech, 7964 Arjons Drive Suite G, San Diego, CA 92126, USA \\ 6. Department of Chemistry and Physics, Louisiana State University, Shreveport, LA 71115, USA \\ E-mail: jaetae.seo@hamptonu.edu
}

Received: 1 August 2020; Accepted: 24 August 2020; Available online: 15 October 2020

\begin{abstract}
The broadband photoluminescence (PL) emissions from CdSe QDs and plasmon-coupled QDs were characterized with time-resolved and temperature-dependent spectroscopy for the application of solid-state white light. The origin of broad spectral emission includes the transitions from bandedge and surface-trapped states. The emission intensity enhancement of plasmon-coupled QDs with respect to that of bare QDs is attributable to the reduction of nonradiative decay and the local field enhancement with plasmon-exciton coupling through the Coulomb interaction. The temperature-dependent and time-resolved PL spectroscopy revealed the existence of selective contribution strength of both the local field enhancement and the reduction of nonradiative decay with plasmon-exciton coupling at different spectral regions.
\end{abstract}

Keywords: Time-resolved spectroscopy; Temperature-dependent; Plasmon-coupled quantum dots.

\section{Introduction}

Semiconductor quantum dots (QDs) have been of great interest for optoelectronic and biomedical applications due to wide optical tunability, high color purity, and high luminescence efficiency [1-5]. The colloidal QDs have characteristic properties of quantum confinement of carriers and a high surface-to-volume ratio that provides sizedependent optical features. The optical features of QDs with dominant surface defects are related to the emission from the bandedge and surface-trapped state transitions. The photoluminescence (PL) from the bandedge transition has relatively narrow spectral width at shorter wavelength, but that of the surface-trapped state is spectrally broad at longer wavelengths. The origin of the surface-trapped state possibly stems from dangling atoms and partial crystallization on the surface of the QDs during the synthesis process. Therefore, the smaller sized nanocrystals possess a higher defect density where the surface-trapped states are located just below the bandedge [6-8]. In some cases, the surface defects are passivated with ligands, such as trioctylphosphine oxide (TOPO) or trioctylphosphine (TOP), or capped with higher bandgap semiconductor ZnS known as a "shell” of the CdSe "core". The surface passivation or capping reduces the spectral width of the bandedge transition, enhances quantum efficiency, and prevents energy transitions from the surface-trapped state. An inorganic shell or organic ligand cap can stabilize the QDs, but may narrow or widen the spectral width [9-12]. The encapsulation with silica or polymer has also been shown to stabilize the QDs [3,13,14]. Without the ligands or shells on semiconductor QDs, the PL distributions and the temperature-sensitive PL intensities are similar to a triplet state in addition to a singlet state in organic materials or semiconductors $[15,16]$. The transitions from surface-trapped states have relatively broad emission at longer spectral regions compared to the emission from the band gap transition, however, with ligands or shells, the QDs may maintain a pure bandedge transition from the lowest level of the conduction band to the highest level of the valence band [4, 17]. In the semiconductor QD community, the reduction of surface-trapped states and nonradiative decay has been pursued in order to achieve better quantum efficiency as well as higher color purity [18-21]. In the effort to remove broadband emission from QDs, the multi-transition surface-trapped state has been overlooked. The broadband spectra that is related to the surface-trapped state may be composed of 
multiple transitions including the surface-trapped state below the conduction band to the highest energy state of valence band (defect-band); the lowest energy state of the conduction band to surface-trapped state above the valence band (band-defect); and/or between the surface-trapped states below the conduction band and above the valence band (defect-defect) [22]. The fractional contribution and the spectral distribution of PL from bandedge transitions and surface-trapped states may be elucidated with time-resolved and temperature-dependent spectroscopy with/without plasmon-exciton coupling through the Coulomb interaction at multiple spectral regions. Another important factor for photonic applications is the quantum yield of the QDs which is enhanced when excitons are coupled with plasmons in metallic nanoparticles (NPs). For LEDs, a high quantum yield is essential to produce high brightness with efficient radiative combination; however, since the broad emission comes from the bandedge and surface-trapped state transitions, nonradiative decay often reduces the PL intensity of the entire spectrum. Instead of surface passivation, which would reduce the surface-trapped state contribution, the use of plasmonic metal nanoparticles for PL enhancement is considered to increase the quantum yield considerably. Previous reports have documented the plasmonic effects on semiconductor QDs, [4, 23, 24, 33] but the fractional contribution of temporal distributions and thermalization of the plasmon-coupled surface-trapped excitons has not been presented in full detail. Plasmonic NPs may reduce the nonradiative decay rate of semiconductor QDs when the plasmon-exciton coupling is much faster than the radiative and nonradiative decay of the QDs [4]. The total PL decay rate of plasmon-coupled QDs may be larger than the PL decay rate of bare QDs without plasmonic coupling because the plasmon-exciton coupling rate in plasmon-coupled QDs competes with the radiative and nonradiative decay rates. It is also possible that some nonradiative energy transfer occurs between the QDs and plasmonic nanoparticles which may decrease the radiative decay rate [25, 26].

Therefore, the plasmon-coupled QDs were characterized using time-resolved and temperature-dependent spectroscopy. The origin of broadband emission including the narrow band emission at $\sim 500 \mathrm{~nm}$ and the wide band emission at $\sim 625 \mathrm{~nm}$ are assigned to the bandedge and surface-trapped state transitions, respectively. Temperature-dependent spectroscopy further clarifies the PL enhancement mechanism for the plasmon-coupled CdSe QDs. The time-resolved spectroscopy yields the PL lifetimes and the fractional contributions of the bandedge transition and surface-trapped state transition to the PL in the presence of plasmonic NPs.

\section{Experimental details}

The CdSe QDs were prepared in a similar fashion to the literature procedure [4]. $6 \mathrm{mmol}$ of CdO and $24 \mathrm{mmol}$ of stearate acid were placed in a 500-ml 3-neck flask and heated until a clear solution was produced (temperature $<200^{\circ} \mathrm{C}$ ). Once cooled down to room temperature, $15 \mathrm{~g}$ of TOPO (99\%), $15 \mathrm{~g}$ of hexadecylamine (95\%), and $80 \mathrm{~g}$ of ODE was added and then heated again. When the temperature reached $280{ }^{\circ} \mathrm{C}$, the heating was stopped and a $40 \mathrm{~g}$ solution (contains $28.5 \mathrm{~g}$ 10\% Se-TOP, $0.5 \mathrm{~g}$ toluene, $11 \mathrm{~g}$ ODE) was swiftly injected. Then, QDs of different sizes were collected at different crystallization times. The shorter (longer) crystallization time provided the smaller (larger) sized QDs near the bulk exciton Bohr radius. The gold (Au) nanoparticles used for plasmonic material were prepared by sodium citrate reduction [27, 28] A solution of HAuCl4 (1mM) in de-ionized water $(550 \mathrm{ml})$ was refluxed with vigorous stirring for $\sim 30$ minutes while the addition of Sodium Citrate was rapidly stirred in for an additional 30 minutes. Au nanoparticles of $\sim 30 \mathrm{~nm}$ diameter were prepared, filtered through $0.45 \mu \mathrm{m}$ polymer membrane filters, and then centrifuged and rinsed.

The absorption spectra of CdSe QDs and Au nanoparticles were measured separately with a UV-VIS spectrometer (Agilent 8453). The PL at room temperature was detected using an optical fiber (Ocean Optics, P600VIS-NIR) and a spectrometer (Ocean Optics, USB4000) with a spectral resolution of $\sim 1 \mathrm{~nm}$. The excitation source was a HeCd laser operating at $442 \mathrm{~nm}$ with a laser power of $\sim 32 \mathrm{~mW}$ through a beam chopper at a frequency of $300 \mathrm{~Hz}$. For the PL measurement, a long wavelength pass (LWP) filter at $\sim 450 \mathrm{~nm}$ was used to block the excitation laser. The temperature-dependent PL was measured using a similar method as the room temperature PL; however, the QDs were dried on quartz micro-glass and placed on the cold finger in an optical cryogenic dewar. The cryogenic dewar consisted of a helium closed-cycle cryostat (Janis, SHI-4-1) equipped with a He-compressor unit (Sumitomo, CNA-11 C) and a temperature controller (Lakeshore, 331). The PL of CdSe QDs with/without plasmon coupling was measured at temperature ranges of $\sim 6 \mathrm{~K}$ to $\sim 300 \mathrm{~K}$.

The time-resolved PL from QDs with/without plasmon coupling were measured by a FluoTime 200 fluorometer (PicoQuant, Inc.) and a diode laser excitation at $\sim 470 \mathrm{~nm}$ with a pulse width of $\sim 120 \mathrm{ps}$ and a repetition rate or $100 \mathrm{KHz}$. The selected wavelengths of PL lifetime measurement were $500 \mathrm{~nm}, 560 \mathrm{~nm}, 625 \mathrm{~nm}$ and $710 \mathrm{~nm}$ for CdSe QDs with/without plasmon coupling to analyze the fractional contribution of transitions from the bandedge and the surface-trapped states. The lifetimes of CdSe QDs and plasmon-coupled QDs were analyzed using a tail fitting with multi-exponential decay equation, and a nonlinear least square function for the best fitting which were described in the literature by Seo et al [29] using the FluoFit4 program (PicoQuant, Inc.). 


\section{Results and discussion}

Figure 1 displays the absorption spectra of CdSe QDs and Au NPs. The absorption peak of Au NPs at $532 \mathrm{~nm}$ is the result of the localized surface plasmon resonance which is attributable to the intraband transitions. The concentration of Au NPs was calculated to be approximately $2.15 \times 10^{-10} \mathrm{~mol} / \mathrm{L}$ by fitting the Mie-scattering theory to the absorption spectrum. The first absorption peak of CdSe materials is located at $\sim 460 \mathrm{~nm}$ indicating the confinement of electrons due to the size reduction of the QDs near the bulk exciton Bohr radius of $\sim 5.8 \mathrm{~nm}$. The large blue-shift of the absorption peak from the bulk at $712 \mathrm{~nm}$ and the multiple discrete absorption peaks provide good evidence for exciton confinement within the dot boundary. The average size of QDs was estimated to be $\sim 2$ $\mathrm{nm}$ using the spectral position of the first absorption peak and the literature information described by Yu et al [1, 2]. The PL spectra exhibit very broad and atypical shape for CdSe QDs which is attributable to the carrier recombination with transitions from both bandedge and surface-trapped states. The first PL peak at $~ 500 \mathrm{~nm}$ is the characteristic bandedge transition which has high color purity and is similar to the PL of previously studied highquality nanocrystals. The second broad emission peak at $\sim 625 \mathrm{~nm}$ is assigned to surface-trapped state transitions which originate from the incomplete crystallization on the surface of the nanocrystals. When the QDs are collected before complete crystallization is achieved, a high density of dangling or incomplete bonds may form on the outer surface of QDs. These dangling or incomplete bonds are known as defects in the crystal structure which form the defect-related surface-trapped states below the lowest level of the conduction band or above the highest level of the valence band. Figure 1 also shows the PL enhancement of plasmon-coupled QDs due to the large local field enhancement and the reduction of nonradiative decay through the effective Coulomb coupling between plasmon and exciton [24]. The broadband emission is distinguishable from typical room light which is produced by the composition of discrete line spectra of Hg-containing fluorescent lamps. Some possible radiative processes may include the bandedge transition between the lowest level of conduction band and the highest level of valence band, the bandedge to deep surface-trapped states, the surface-trapped to the valence band, and shallow surface-trapped state to deep surface-trapped state. Therefore, the time-resolved PL intensity decays at different wavelengths of $500 \mathrm{~nm}, 560 \mathrm{~nm}, 625 \mathrm{~nm}$, and $710 \mathrm{~nm}$ were analyzed to characterize the relative fractional contributions of each transition to their respective spectral regions.

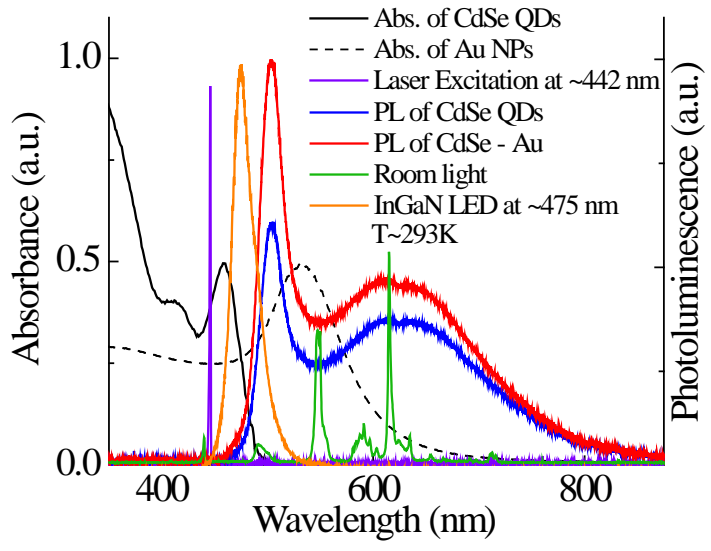

Fig. 1. Absorption spectra of Au, Absorption and PL of QD with/without plasmon coupling, the spectra of laser excitation source and InGaN, and the spectrum of room light (typical fluorescent lamp).

In addition, temperature-dependent spectroscopy of CdSe QDs and plasmon-coupled QDs was performed to investigate the thermal quenching at the bandedge and surface-trapped states as well as the potential thermalization from the surface-trapped state to the bandedge. The temperature-dependent PL of (a) CdSe QDs and (b) plasmoncoupled QDs are shown in figure 2. The optical transition includes both radiative and nonradiative transitions. The nonradiative transition generally increases as the temperature increases due to exciton-phonon, -defect, and/or impurity scatterings in semiconductors [30]. Figure 2 (a) shows that the PL at surface-trapped state is increased $\sim 6$-fold at $6 \mathrm{~K}$ compared to $300 \mathrm{~K}$. The $\sim 6$-fold increase at surface-trapped state at $6 \mathrm{~K}$ compared to $300 \mathrm{~K}$ can be attributed to the reduction of nonradiative transitions through exciton-phonon, -defect, and/or -impurity scatterings. Figure 2 (a) also shows that the PL at the bandedge is relatively stable and thus temperature-independent in the range of $300 \mathrm{~K}$ to $6 \mathrm{~K}$. The thermal quenching of PL intensity at the bandedge was expected for QDs with neither surface passivation nor plasmon-coupling due to the increased likelihood of nonradiative transitions at higher temperatures. The observations of strong thermal quenching of PL intensity at the bandedge were reported for ligand-passivated QDs or coreshells at higher temperatures. It implies the existence of an exciton thermalization 
mechanism [31, 32] from the surface-trapped state to the bandedge at higher temperature. Figure 2 (b) shows the temperature-dependent PL of QDs with plasmon coupling. The PL intensity at the bandedge was increased 2fold while the surface-trapped state was increased $\sim 1.5$-fold at $6 \mathrm{~K}$ compared to $300 \mathrm{~K}$. The smaller thermal quenching at the surface-trapped states and the appearance of thermal quenching at bandedge for the plasmoncoupled QDs, compared to bare QDs, indicates that the plasmon-exciton coupling rate is much higher than the nonradiative decay rate and that thermalization from the surface-trapped state to the bandedge occurs within this temperature range. Therefore, the PL intensity of plasmon-coupled QD is mainly accredited to local field enhancement and exciton-plasmon coupling. In addition, the time-resolved PL spectroscopy provides a better description on the contributions of plasmon-exciton coupling rate and/or local field enhancement to PL lifetimes and fractional amplitudes at different spectral regions.
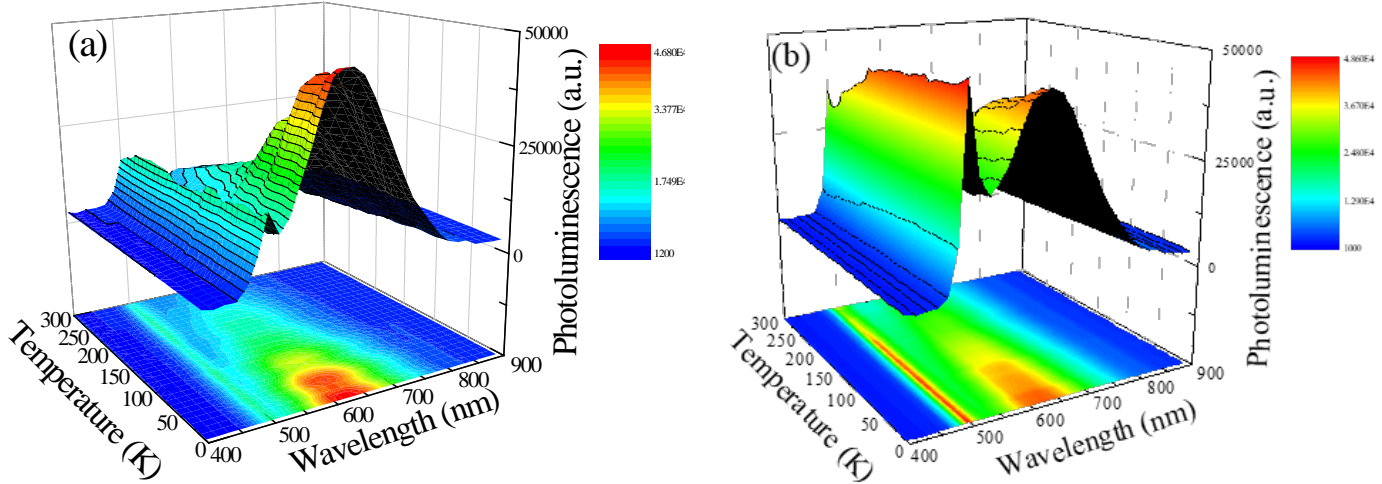

Fig. 2. Temperature-dependent PLs of (a) CdSe QDs and (b) plasmon-coupled CdSe QDs.

The time-resolved spectroscopy at different spectral regions elucidates the temporal dynamics of PL at the bandedge and surface-trapped states, and provides a deeper understanding of broadband emissions from plasmoncoupled QDs. Figure 3 shows the time-resolved PL of CdSe QDs and plasmon-coupled CdSe QDs at (a) $500 \mathrm{~nm}$, (b) $560 \mathrm{~nm}$, (c) $625 \mathrm{~nm}$, and (d) $710 \mathrm{~nm}$. The fractional amplitudes, three exponential decay components, and averaged PL lifetimes of QD and plasmon-coupled QDs are listed in table 1. CdSe QD emission may include transitions from bandedge to valence band (interband), bandedge to valence band trapped state (band-defect), surface-trapped state to valence band (defect-band), and the surface-trapped state just below the conduction band to the trapped state just above the valence band (defect-defect). The fast $\left(\tau_{1}\right)$, intermediate $\left(\tau_{2}\right)$, and slow $\left(\tau_{3}\right)$ lifetimes are assigned to the interband, defect-band / band-defect, and defect-defect transitions, respectively.

The fractional amplitude [4, 24] changes of the fast PL lifetime $\tau_{1}$ between QDs and plasmon-coupled QDs are nearly negligible as shown in figure 3 . The amplitudes of $\tau_{1}$ changed from $58.17 \%$ to $57.46 \%$ at $500 \mathrm{~nm}, 70.73 \%$ to $67.15 \%$ at $560 \mathrm{~nm}, 59.35 \%$ to $56.01 \%$ at $625 \mathrm{~nm}$, and $40.64 \%$ to $38.55 \%$ at $710 \mathrm{~nm}$. The fractional amplitude contributions of $\tau_{1}$ to the average PL lifetime reduced at longer wavelengths because the amplitude contribution of $\tau_{3}$ to the average PL lifetime increased. The PL lifetimes, $\tau_{1}$, of plasmon-coupled QDs with respect to $\tau 1$ of bare QDs at $\sim 500 \mathrm{~nm}, \sim 560 \mathrm{~nm}$, and $\sim 710 \mathrm{~nm}$ were shortened from $11.05 \mathrm{~ns}$ to $4.49 \mathrm{~ns}, 4.57 \mathrm{~ns}$ to $3.85 \mathrm{~ns}$, and 17.65 ns to $6.07 \mathrm{~ns}$, respectively. The PL lifetime $\tau_{1}$ of plasmon-coupled QDs with respect to $\tau_{1}$ of bare QDs at $\sim 625 \mathrm{~nm}$ was lengthened from 5.94 ns to 6.81 ns likely due to the high density of defect-related excitons at the spectral peak. The fractional amplitudes for the intermediate PL lifetime, $\tau_{2}$, of plasmon-coupled QDs with respect to the $\tau 2$ amplitudes of bare QDs were changed from $34.72 \%$ to $34.29 \%$ at $500 \mathrm{~nm}, 21.04 \%$ to $24.72 \%$ at $560 \mathrm{~nm}, 26.81 \%$ to $24.86 \%$ at $625 \mathrm{~nm}$, and $37.74 \%$ to $31.86 \%$ at $710 \mathrm{~nm}$. The PL lifetimes, $\tau_{2}$, of plasmon-coupled QDs with respect to $\tau_{2}$ of bare QDs at $\sim 500 \mathrm{~nm}, \sim 560 \mathrm{~nm}, \sim 625 \mathrm{~nm}$ and $\sim 710 \mathrm{~nm}$ were shortened from $55.26 \mathrm{~ns}$ to $22.76 \mathrm{~ns}$, $51.25 \mathrm{~ns}$ to $24.19 \mathrm{~ns}, 71.67 \mathrm{~ns}$ to $53.4 \mathrm{~ns}$, and $115.01 \mathrm{~ns}$ to $54.7 \mathrm{~ns}$, respectively. This result indicates an increased radiative decay rate due to plasmon-exciton coupling at the band-defect / defect-band transitions.

The fractional amplitudes for the slow PL lifetime, $\tau_{3}$, of plasmon-coupled QDs with respect to $\tau_{3}$ of bare QDs were changed from $7.12 \%$ to $8.25 \%$ at $500 \mathrm{~nm}, 8.24 \%$ to $8.13 \%$ at $560 \mathrm{~nm}, 13.84 \%$ to $19.13 \%$ at $625 \mathrm{~nm}$, and $21.62 \%$ to $29.58 \%$ at $710 \mathrm{~nm}$. The PL lifetimes, $\tau_{3}$, of plasmon-coupled QDs with respect to $\tau_{3}$ of bare QDs at $\sim 500$ $\mathrm{nm}$ and $\sim 560 \mathrm{~nm}$ were shortened from $212.19 \mathrm{~ns}$ to $114.35 \mathrm{~ns}$ and $254.1 \mathrm{~ns}$ to $202.0 \mathrm{~ns}$, respectively. However, the PL lifetimes, $\tau_{3}$, of plasmon-coupled QDs with respect to $\tau_{3}$ of bare QDs at $\sim 625 \mathrm{~nm}$ and $\sim 710 \mathrm{~nm}$ were lengthened from 318.74 ns to 392.1 ns and 402.4 ns to 416.4 ns, respectively. The longer PL lifetimes of plasmon-coupled QDs compared to bare QDs are attributable to the local field enhancement rather than the reduction of nonradiative decay rate due to plasmon-exciton coupling.

The average PL lifetime of plasmon-coupled QDs with respect to bare QDs at $500 \mathrm{~nm}$ and $560 \mathrm{~nm}$ was shortened from $40.71 \mathrm{~ns}$ to $19.82 \mathrm{~ns}$ and $34.95 \mathrm{~ns}$ to $25.0 \mathrm{~ns}$, respectively. This indicates that the average PL 
lifetime of plasmon-coupled QDs with respect to bare QDs at shorter wavelengths is attributable to the reduction of nonradiative decay due to plasmon-exciton coupling. However, the average PL lifetime of plasmon-coupled QDs at $\sim 625 \mathrm{~nm}$ and $\sim 710 \mathrm{~nm}$ was lengthened from $66.87 \mathrm{~ns}$ to $92.1 \mathrm{~ns}$ and $137.6 \mathrm{~ns}$ to 142.95 ns, respectively, compared to bare QDs. This indicates that the longer average exciton lifetime of plasmon-coupled QDs with respect to bare QDs at longer wavelengths is accredited to the local field enhancement rather than the reduction of the nonradiative decay rate due to plasmon-exciton coupling rates. Therefore, the broad emission of plasmoncoupled QDs is enhanced by selective contribution strength between nonradiative decay reduction due to the plasmon-exciton coupling rate at shorter wavelengths and local field enhancement at longer wavelengths.
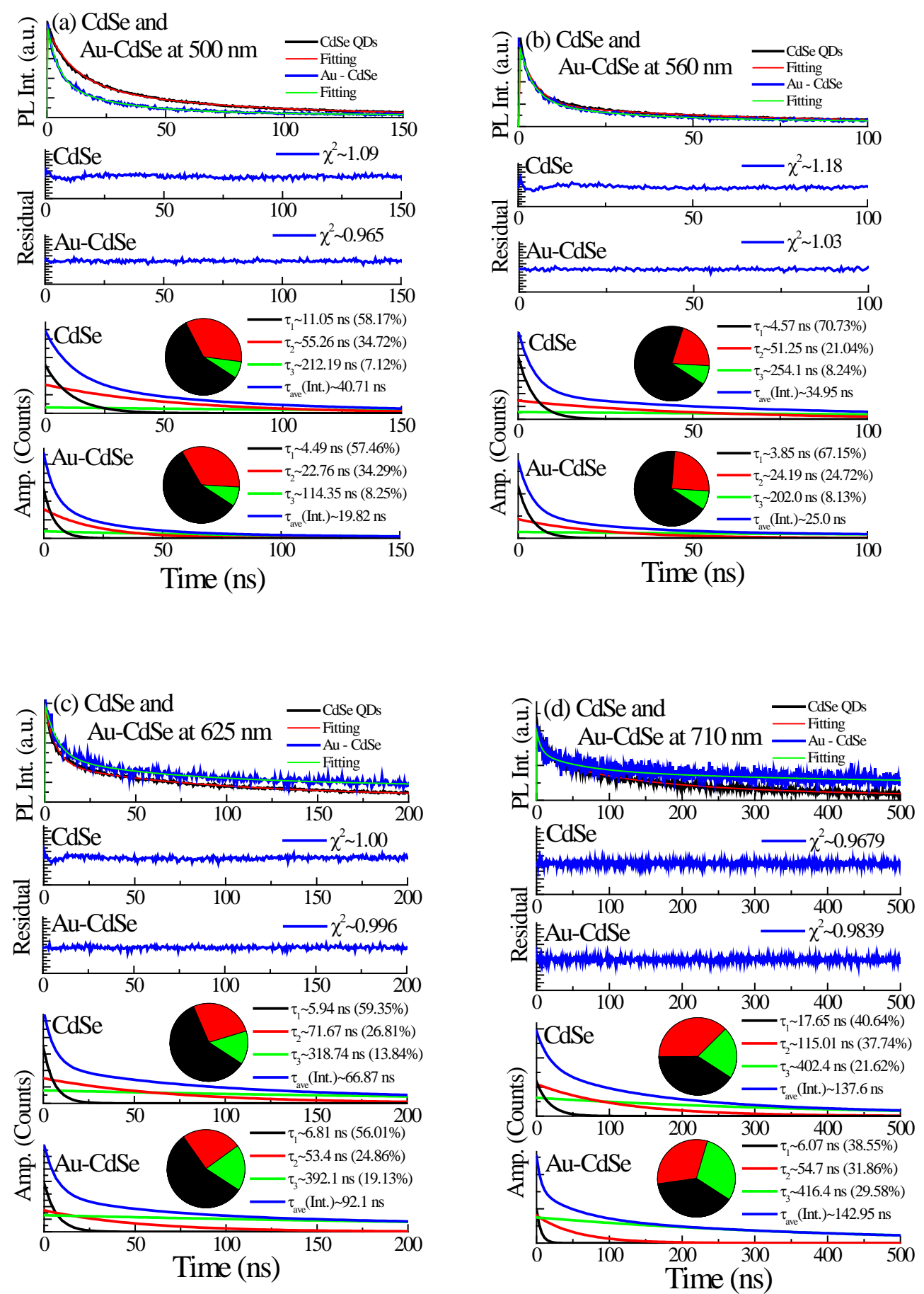

Fig. 3. Time-resolved PL of CdSe QDs and plasmon-coupled CdSe QDs at (a) $500 \mathrm{~nm}$, (b) $560 \mathrm{~nm}$, (c) $625 \mathrm{~nm}$, and (d) $710 \mathrm{~nm}$. 
Table 1. Lifetime and fractional amplitudes of CdSe QDs (top) and plasmon-coupled CdSe QDs (bottom).

\begin{tabular}{|c|c|c|c|c|}
\hline CdSe & $500 \mathrm{~nm}$ & $560 \mathrm{~nm}$ & $625 \mathrm{~nm}$ & $710 \mathrm{~nm}$ \\
\hline$\tau_{1}$ & $\begin{array}{l}11.05 \mathrm{~ns} \\
(58.17 \%)\end{array}$ & $\begin{array}{l}4.57 \mathrm{~ns} \\
(70.73 \%)\end{array}$ & $\begin{array}{l}5.94 \mathrm{~ns} \\
(59.35 \%)\end{array}$ & $\begin{array}{l}17.65 \mathrm{~ns} \\
(40.64 \%)\end{array}$ \\
\hline$\tau_{2}$ & $\begin{array}{l}55.26 \mathrm{~ns} \\
(34.72 \%)\end{array}$ & $\begin{array}{l}51.25 \mathrm{~ns} \\
(21.04 \%)\end{array}$ & $\begin{array}{l}71.67 \mathrm{~ns} \\
(26.81 \%)\end{array}$ & $\begin{array}{l}115.01 \mathrm{~ns} \\
(37.74 \%)\end{array}$ \\
\hline$\tau_{3}$ & $\begin{array}{l}212.19 \mathrm{~ns} \\
(7.12 \%)\end{array}$ & $\begin{array}{l}254.1 \mathrm{~ns} \\
(8.24 \%)\end{array}$ & $\begin{array}{l}318.74 \text { ns } \\
(13.84 \%)\end{array}$ & $\begin{array}{l}402.4 \mathrm{~ns} \\
(21.62 \%)\end{array}$ \\
\hline$\tau_{\text {ave }}$ & $40.71 \mathrm{~ns}$ & $34.95 \mathrm{~ns}$ & $66.87 \mathrm{~ns}$ & $137.6 \mathrm{~ns}$ \\
\hline $\mathrm{Au}-\mathrm{CdSe}$ & $500 \mathrm{~nm}$ & $560 \mathrm{~nm}$ & $625 \mathrm{~nm}$ & $710 \mathrm{~nm}$ \\
\hline$\tau_{1}$ & $\begin{array}{l}4.49 \mathrm{~ns} \\
(57.46 \%)\end{array}$ & $\begin{array}{l}3.85 \mathrm{~ns} \\
(67.15 \%)\end{array}$ & $\begin{array}{l}6.81 \mathrm{~ns} \\
(56.01 \%)\end{array}$ & $\begin{array}{l}6.07 \mathrm{~ns} \\
(38.55 \%)\end{array}$ \\
\hline$\tau_{2}$ & $\begin{array}{l}22.76 \mathrm{~ns} \\
(34.29 \%)\end{array}$ & $\begin{array}{l}24.19 \mathrm{~ns} \\
(24.72 \%)\end{array}$ & $\begin{array}{l}53.4 \mathrm{~ns} \\
(24.86 \%)\end{array}$ & $\begin{array}{l}54.7 \mathrm{~ns} \\
(31.86 \%)\end{array}$ \\
\hline$\tau_{3}$ & $\begin{array}{l}114.35 \mathrm{~ns} \\
(8.25 \%)\end{array}$ & $\begin{array}{l}202.0 \mathrm{~ns} \\
(8.13 \%)\end{array}$ & $\begin{array}{l}392.1 \mathrm{~ns} \\
(19.13 \%)\end{array}$ & $\begin{array}{l}416.4 \mathrm{~ns} \\
\text { (29.58\%) }\end{array}$ \\
\hline$\tau_{\mathrm{ave}}$ & $19.82 \mathrm{~ns}$ & $25.0 \mathrm{~ns}$ & $92.1 \mathrm{~ns}$ & $142.95 \mathrm{~ns}$ \\
\hline
\end{tabular}

\section{Conclusion}

The broadband PL emissions from CdSe QDs and plasmon-coupled QDs were characterized with time-resolved and temperature-dependent spectroscopy for the application of solid-state white light. The origin of broad spectral emission includes the transitions from the bandedge and surface-trapped states. The temperature-dependent PL spectroscopy of CdSe QDs and plasmon-coupled CdSe QDs were characterized by the thermal quenching of PL intensity at the bandedge and surface-trapped states, in addition to the thermalization from the surface-trapped state to the bandedge. The observation of negligible thermal quenching of PL intensity from CdSe QDs at the bandedge implies the existence of an exciton thermalization mechanism from the surface-trapped state to the bandedge at higher temperature. The miniscule thermal quenching at the surface-trapped states in conjunction with the appearance of thermal quenching at the bandedge for the plasmon-coupled QDs indicates the selective contribution between nonradiative decay reduction due to the plasmon-exciton coupling rate at shorter wavelengths and local field enhancement at longer wavelengths. The average PL lifetime of plasmon-coupled QDs at shorter (longer) wavelengths was shorter (longer) than bare QDs, while the PL intensity of plasmon-coupled QDs was enhanced at both shorter and longer wavelengths compared to QDs. This indicates that the selective contribution strength between nonradiative decay reduction due to the plasmon-exciton coupling rate at shorter wavelengths and local field enhancement to the average PL lifetime at longer wavelengths. Therefore, both the temperature-dependent and the time-resolved PL spectroscopy identically conclude the selective contribution strength of both plasmon-exciton coupling and large local field enhancement to the increased broadband PL intensity at different spectral regions.

\section{Acknowledgement}

The work at HU was supported by ARO W911NF-15-1-0535, NASA NNX15AQ03A, and NSF HRD-1137747.

\section{References}

[1] Yu W, Qu L, Guo W, Peng X. Experimental determination of the extinction coefficient of CdTe, CdSe, and CdS nanocrystals. Chemistry of Materials. 2003;15:2854-2860.

[2] Yu W, Qu L, Guo W, Peng X. Experimental determination of the extinction coefficient of CdTe, CdSe, and CdS nanocrystals: correction. Chemistry of Materials. 2004;16:560.

[3] Kim S, Seo J, Ramdon R, Pyo HB, Song K, Kang BH. Solid-phase immunoassay of polystyrene-encapsulated semiconductor coreshells for cardiac marker detection. Journal of Nanomaterials. 2012;2012:693575

[4] Seo J, Fudala R, Kim WJ, Rich R, Tabibi B, Cho H, Gryczynski Z, Gryczynski I, Yu W. Hybrid optical materials of plasmon-coupled CdSe/ZnS coreshells for photonic applications. Optical Materials Express. 2012;2:1026-1039.

[5] Khan SA, Smith GT, Seo F, Ellerbee AK. Label-free and non-contact optical biosensing of glucose with quantum dots. Biosensensensors and Bioelectronics. 2015;64:30-35.

[6] Giansante C, Infante I. Surface traps in colloidal quantum dots: a combined experimental and theoretical perspective. Journal of Physical Chemistry Letters. 2017;8: 5209-5215. 
[7] Almeida AJ, Sahu A, Riedinger A, Norris DJ, Brandt MS, Stutzmann M, Pereira RN. Charge trapping defects in CdSe nanocrystal quantum dots. Journal of Physical Chemistry C. 2016;120:13763-13770.

[8] Veamatahau A, Jiang B, Seifert T, Makuta S, Latham K, Kanehara M, Teranishi T, Tachibana Y. Origin of surface trap states in CdS quantum dots: relationship between size dependent photoluminescence and sulfur vacancy trap states. Physical Chemistry Chemical Physics. 2015;17:2850-2858.

[9] Liu W, Zhang Y, Ruan C, Wang D, Zhang T, Feng Y, Gao W, Yin J, Wang Y, Riley AP, Hu MZ, Yu WW. ZnCuInS/ZnSe/ZnS quantum dot-based downconversion light-emitting diodes and their thermal effect. Journal of Nanomaterials. 2015;2015:298614.

[10] Zhang Y, Dai Q, Li X, Liang J, Colvin VL, Wang Y, Yu WW. PbSe/CdSe and PbSe/CdSe/ZnSe hierarchical nanocrystals and their photoluminescence. Langmuir. 2011;27:9583-9587.

[11] Zhang Y, Dai Q, Li X, Cui Q, Gu Z, Zou B, Wang Y, Yu WW. Formation of PbSe/CdSe core/shell nanocrystals for stable near-infrared high photoluminescence emission. Nanoscale Research Letters. 2010;5:1279.

[12] Dai Q, Zhang Y, Wang Y, Zou B, Yu WW, Hu MZ. Ligand effects on synthesis and post-synthetic stability of PbSe nanocrystals. Journal of Physical Chemistry C. 2010;114:16160-16167.

[13] Sun C, Zhang Y, Shen X, Wang Y, Chen X, Ji C, Shen H, Shi H, Wang Y, Yu WW. Highly luminescent, stable, transparent and flexible perovskite quantum dot gels towards light-emitting diodes. Nanotechnology. 2017;28:365601.

[14] Sun C, Zhang Y, Ruan C, Yin C, Wang X, Wang Y, Yu WW. Efficient and stable white LED based on silicacoated inorganic perovskite quantum dots. Advanced Materials. 2018;28:10088-10094.

[15] Vlaskin VA, Janssen N, Rijssel JV, Beaulac RM, Gamelin DR. Tunable dual emission in doped semiconductor nanocrystals. Nano Letters. 2010;10:3670-3674.

[16] Parker CA, Hatchard CG. Triplet-singlet emission in fluid solutions. Phosphorescence of eosin. Transactions of the Faraday Society. 1961;57:1894-1904.

[17] Javier A, Magana D, Jennings T, Strouse GF. Nanosecond exciton recombination dynamics in colloidal CdSe quantum dots under ambient conditions. Applied Physics Letters. 2003;83:1423-1425.

[18] Owen J, Brus L. Chemical synthesis and luminescence applications of colloidal semiconductor quantum dots. Journal of the American Chemical Society. 2017:139:10939-10943.

[19] Hollingsworth JA. Nanoscale engineering facilitated by controlled synthesis: From structure to function. Coordination Chemistry Reviews. 2014;263:197-216.

[20] Dabbousi BO, Rodriguez-Viejo J, Mikulec FV, Heine JR, Mattoussi H, Ober R, Jensen KF,Bawendi MG. (CdSe)ZnS core-shell quantum dots: synthesis and characterization of a size series of highly luminescent nanocrystallites. Journal of Physical Chemistry B. 1997;101:9463-9475.

[21] Hines MA, Guyot-Sionnest P. Synthesis and characterization of strongly luminescing ZnS-Capped CdSe nanocrystals. Journal of Physical Chemistry. 1996;100:468-471.

[22] Knowles KE, McArthur EA, Weiss EA. A multi-timescale map of radiative and nonradiative decay pathways for excitons in CdSe quantum dots. ACS Nano. 2011;5:2026-2035.

[23] Rigo MV, Seo J. Probing plasmon polarization-mediated photoluminescence enhancement on metalsemiconductor hybrid optical nanostructures. Chemical Physics Letters. 2011;517:190-195.

[24] Rice Q, Raut S, Chib R, Hayes A, Gryczynski Z, Gryczynski I, Kim YK, Tabibi B, Seo J. Defect-mediated spontaneous emission enhancement of plasmon-coupled CuInS2 and CuInS $2 / Z n S$. Optical Materials Express. 2016;6:566-577.

[25] Kulakovich O, Strekal N, Yaroshevich A, Maskevich S, Gaponenko S, Nabiev I, Woggon U, Artemyev M. Enhanced luminescence of CdSe quantum dots on gold colloids. Nano Letters. 2002;2:1449-1452.

[26] Song JH, Atay T, Shi S, Urabe H, Nurmikko AV. Large ehancement of fluorescence efficiency from $\mathrm{CdSe} / \mathrm{ZnS}$ quantum dots induced by resonant coupling to spatially controlled surface plasmons. Nano Letters. 2005;5:1557-1561.

[27] Seo J, Yang Q, Kim W, Heo J, Ma S, Austin J, Yun WS, Jung SS, Han SW, Tabibi B, Temple D. Optical nonlinearities of Au nanoparticles and Au/Ag coreshells. Optics Letters. 2009;34:307-309.

[28] Kim W, Seo J, Austin J, Yun WS, Ah CS, Sung GY, Choi IS. Colorimetric analysis on flocculation of bioinspired Au self-assembly for bio-photonic application. Journal of Nanomaterials. 2009;2009:261261.

[29] Seo J, Raut S, Abdel-Fattah M, Rice Q, Tabibi B, Rich R, Fudala R, Gryczynski I, Gryczynski Z, Kim W J, Jung S, Hyun R. Time-resolved and temperature-dependent photoluminescence of ternary and quaternary nanocrystals of CuInS 2 with ZnS capping and cation exchange. Journal of Applied Physics. 2013;114:094310.

[30] Kohler J, Ashida M, Kato R, Schmidt T, Schmidt D. Slow thermalization of singlet excitons in $\mathrm{NaNO}_{2}$ crystals. Journal of Luminescence. 1991;47:239-248.

[31] Seo JT, Hommerich UH, Steckl AJ, Birkhahn R, Zavada JM. Green luminescence and excited state thermalization in Er-doped gallium nitride. Journal of the Korean Physical Socety. 2006;49:943-946. 
[32] Hömmerich U, Seo JT, Abernathy CR, Steckl AJ, Zavada JM. Spectroscopic studies of the visible and infrared luminescence from Er doped GaN. Material Science and Engineering B. 2001;81:116-120.

[33] Liu E, Zhu H, Yi J, Kobbekaduwa K, Adhikari P, Liu J, Shi Y, Zhang J, Li H, Oprisan A, Rao AM, Sanabria $\mathrm{H}$, Chen O, Gao J. Manipulating charge transfer from core to shell in CdSe/CdS/Au heterojunction quantum dots. ACS Applied Materials \& Interfaces. 2019;11(51): 48551-48555.

(C) 2020 by the author(s). This work is licensed under a Creative Commons Attribution 4.0 International License (http://creativecommons.org/licenses/by/4.0/). Authors retain copyright of their work, with first publication rights granted to Tech Reviews Ltd. 